\title{
Use cases for genetic epidemiology in malaria elimination
}

\author{
Ronit Dalmat ${ }^{1,4+}$, Brienna Naughton ${ }^{2,4+}$, Tao Sheng Kwan-Gett ${ }^{3,4}$, Jennifer Slyker ${ }^{1,2,4}$ and Erin M. Stuckey $5^{*^{*}}$
}

\begin{abstract}
Background: While traditional epidemiological approaches have supported significant reductions in malaria incidence across many countries, higher resolution information about local and regional malaria epidemiology will be needed to efficiently target interventions for elimination. The application of genetic epidemiological methods for the analysis of parasite genetics has, thus far, primarily been confined to research settings. To illustrate how these technical methods can be used to advance programmatic and operational needs of National Malaria Control Programmes (NMCPs), and accelerate global progress to eradication, this manuscript presents seven use cases for which genetic epidemiology approaches to parasite genetic data are informative to the decision-making of NMCPS.
\end{abstract}

Methods: The use cases were developed through a highly iterative process that included an extensive review of the literature and global guidance documents, including the 2017 World Health Organization's Framework for Malaria Elimination, and collection of stakeholder input. Semi-structured interviews were conducted with programmatic and technical experts about the needs and opportunities for genetic epidemiology methods in malaria elimination.

Results: Seven use cases were developed: Detect resistance, Assess drug resistance gene flow, Assess transmission intensity, Identify foci, Determine connectivity of parasite populations, Identify imported cases, and Characterize local transmission chains. The method currently used to provide the information sought, population unit for implementation, the pre-conditions for using these approaches, and post-conditions intended as a product of the use case were identified for each use case.

Discussion: This framework of use cases will prioritize research and development of genetic epidemiology methods that best achieve the goals of NMCPs, and ultimately, inform the establishment of normative policy guidance for their uses. With significant engagement of stakeholders from malaria endemic countries and collaboration with local programme experts to ensure strategic implementation, genetic epidemiological approaches have tremendous potential to accelerate global malaria elimination efforts.

Keywords: Malaria, Use case, Genetic epidemiology, Drug resistance, NMCP, Gene flow, Eradication, Surveillance, Policy development, Transmission

\section{Background}

In recent years, public health campaigns have achieved major reductions in malaria morbidity and mortality globally, with incidence decreasing by $18 \%$ globally between 2010 and 2016 [1].

As many countries accelerate their efforts towards elimination (World Health Organization (WHO) E-2020

\footnotetext{
*Correspondence: erin.stuckey@gatesfoundation.org

${ }^{\dagger}$ Ronit Dalmat and Brienna Naughton contributed equally to this work

${ }^{5}$ Bill and Melinda Gates Foundation, Seattle, WA, USA

Full list of author information is available at the end of the article
}

Initiative) and the world works towards global eradication (Sustainable Development Goal (SDG) 3.3), programmatic priorities are changing and new data needs emerging. Traditional epidemiological approaches to surveillance analysing the reported clinical-based incidence data available through the routing system and/ or cross-sectional studies of prevalence in a population were previously sufficient for National Malaria Control Programmes (NMCPs), whose aim is to contain and estimate the burden of infection within its borders. However, higher resolution information-i.e., more detailed, comprehensive, accurate, and informative-about parasite 
transmission dynamics will be necessary to target interventions or even predict when, where, and which interventions will be most effective to eliminating remaining reservoirs of infection.

Genetic epidemiology is a discipline that deals with the role of genetic factors and their interaction with environmental factors in the occurrence of disease in populations [2]. Analysing parasite genetics has enormous potential to aid both NMCP efforts at elimination as well as international efforts toward eradication [3, 4]. To date the genetic epidemiology field has primarily focused on applied research. For example, the use of molecular markers of anti-malarial drug resistance has for some time been used to guide the efficacy studies that define treatment policy; more recently, the detection of parasites mutant for histidine-rich protein $2 / 3$ (pfhrp2/pfhrp3) gene highlighted the need to develop rapid diagnostic tests (RDTs) with alternative targets of detection, and to provide countries with guidance on the implications for case management [5]. A global scientific network providing framework for generating, integrating and sharing malaria parasite and vector genetic and genomic data is available through the Malaria Genomic Epidemiology Network (MalariaGEN) [6].

Given this vision for genetic epidemiology approaches in NMCP elimination strategies and the need for WHO normative policy guidance to effect global implementation, there is an emerging need to clearly articulate the uses of these methods and how they can help advance programmatic and operational needs of NMCPs as well as accelerate progress toward SDG 3.3. To bridge the divide between research and implementation, use cases are a means to align research, technology development, and public health efforts using genetic epidemiology methods in support of NMCP priorities. Use case analysis is an analytic framework commonly employed in software and business industries to assess the ways a system can be used and illustrate its applications in real world contexts [7]. A use case provides a functional description of a system, as well as a framework to develop derivations of test cases based on available technologies. They are then used to inform the application and implementation of new technologies through ongoing engagement with stakeholders. Other health domains have deployed a use case methodology for emerging technologies, including soil-transmitted helminth diagnostics [8] and unmanned aerial vehicles [9]. This report represents a novel application of this methodology to genetic epidemiology and is intended to launch a cohesive collaboration by genetics researchers and public health programmes towards the use of these approaches (Fig. 1).

\section{Methods}

To begin, a review was conducted of literature regarding genetic epidemiology and malaria elimination strategies. The literature review engaged an exploratory search strategy in both published and grey literature databases such as the MESA research hub [10], starting with key search terms, and allowing those findings to inform the next cycle of search terms and contacts in a cascade search. Initial search terms included malaria transmission, genetic epidemiology, genetic sequencing, and population genetics.

To inform and complement the literature review, between March and September 2017, individual in-depth interviews were conducted with 15 international stakeholders identified through the review-including malaria disease experts, laboratory and field researchers, programme implementers, mathematical modellers, policymakers, and donors for their input as to the needs and opportunities for genetic information in malaria elimination. Interviews were conducted in person or over the phone using a semi-structured interview guide (Additional file 1).

The development of the list of use cases was informed by the critical review of the literature and informational interviews with experts. The use cases were aligned to activities specifically described in Pillars 2 and 3 of the updated 2017 WHO Framework for Malaria Elimination [11], stemming from the WHO Global Technical Strategy [12]. These two pillars comprise activities for which genetic epidemiology applications have the most promising applications: surveillance, case investigation, and stratification of areas for targeted interventions to reduce transmission and accelerate elimination. Activities falling outside the WHO framework, for example using genetic techniques to guide product development such as rapid diagnostic tests (RDTs), were considered out of scope. This use case framework is modeled on an early draft of use cases developed by a working group of soil-transmitted helminths diagnostic experts published last year [8]. Finally, the categorization and content of the use cases was iteratively developed and revised by sharing drafts with previously interviewed experts.

\section{Results}

Seven use cases are presented where genetic epidemiology approaches are informative to decision-making within the efforts of NMCPs. The use cases are intended to be technology-agnostic such that they may be generalizable across many endemic settings with different epidemiological characteristics (e.g. disease prevalence, drug resistance incidence, and transmission intensity), as well as applicable to emerging genetic epidemiology methods. 


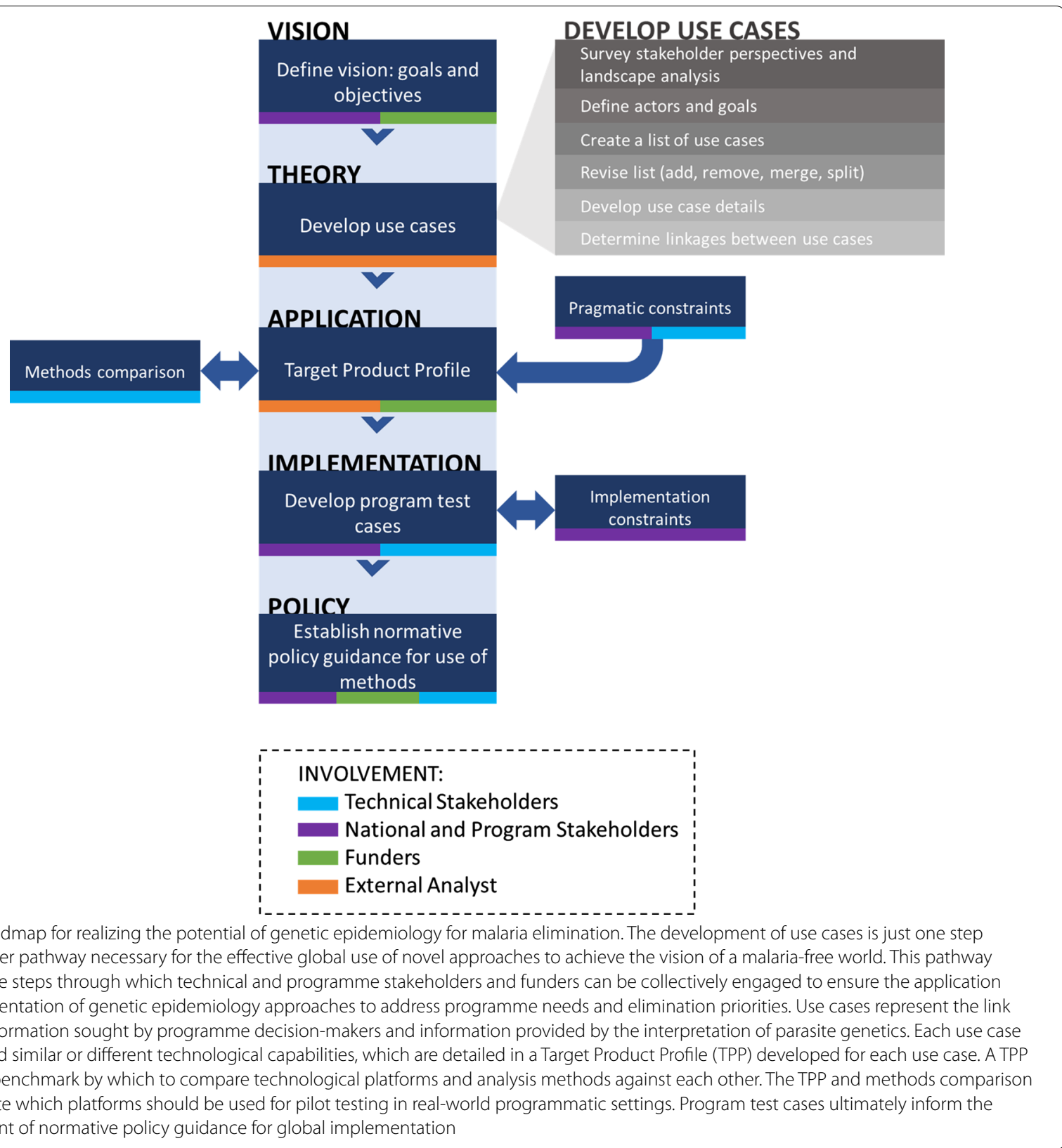

\section{Components of a malaria use case}

Use cases are designed to align multiple scientific fields related to malaria control and eradication under a singular goal, incorporating research and programme perspectives. The structure of a use case delineates the conditions under which the method or tool would be applicable and useful. The content of each use case, presented here, is organized into three components: description, pre-conditions, and post-conditions.

The description section conveys, briefly, the objective sought by use of the method-what information the genetic epidemiology method is providing-as well as the current method used to achieve that objective in the absence of a genetic method. Pre-conditions describe the type of case detection (active, passive, or reactive), prevalence of infection (high, moderate, low, and very low), and focus type (active, residual non-active, and cleared). These categorizations of epidemiological characteristics are all aligned to those used in the WHO Framework for Elimination and use the same definition. Sampling frame (representative or dense) is also listed as a pre-condition to depict the degree to which the target population needs to 
be sampled for a genetic method to be informative. Finally, the population level of implementation (e.g. individual or focus) is described. These vary across use cases and serve to provide an orientation to the objectives. Post-conditions focus on the consumers of information and depict how the data will be identified, utilized, and presented. This includes the potential actions informed by the results of the genetic method, presentation of that output, and the ideal timeframe between sample collection and delivery of data analysis on which the information would be available from the method in order to be informative to programmatic decision-making.

For some of the use cases described here, genetic methods have been applied in a malaria control or elimination setting and described in the literature (Use cases 1,2, and 6); methods for other use cases have not yet been applied programmatically (Use cases 3, 4, 5, and 7). Use cases are provided in Table 1 and described in short narratives below, with one or more example methods, if available.

These use cases are designed to align the efforts of multiple scientific fields towards the shared goal of malaria elimination, incorporating both research and programme perspectives. The content of each use case presents the conditions under which the method or tool is applicable and how it is useful.

\section{Use case \#1: Detect resistance}

This use case describes a method that identifies drug resistant parasites within an individual with high sensitivity and specificity, regardless of the presence of symptoms or treatment failure, in a high to very low prevalence setting. Treatment efficacy surveys are currently used to assess population drug resistance but are time-consuming, costly, and may occur with insufficient frequency to inform decision-making. At a population level, these methods, compared to traditional methods, would serve to estimate resistance prevalence more accurately in a country and accelerate the identification of population trends in the emergence of resistance. Numerous genotyping methods have been developed for detecting particular genetic markers in individual patient samples; nested and real-time polymerase chain reaction (PCR) are used widely in a variety of country settings. Other newer methods still in development include: pointof-care resistance testing with loop-mediated isothermal amplification [13, 14] and next generation sequencing (NGS) of single nucleotide polymorphism (SNPs) [15]. Each approach has advantages and limitations described in the literature [16-20].

\section{Use case \#2: Assess drug resistance gene flow}

The method depicted in this use case provides information about how resistance genes are spreading in a population of parasites in a setting with high to very low prevalence. The information provided by the use case enables identification of linked populations and predictive modelling of drug-based interventions. Methods for population genetic analysis have been employed to understand the interaction between gene flow and patterns of resistant marker prevalence in several geographies including Ethiopia [21] and Cambodia [22], as well as the dispersal of resistance haplotypes in Eastern Africa and the Democratic Republic of Congo [23]. NGS technologies are also emerging as a tool for drug resistance surveillance [24]. This use case is linked to the method described by Use case 1 , as it would require aggregation of information collected by Use case 1 , along with additional analysis to predict emergence or dissemination of drug resistance into new areas. Application of this use case is most applicable at a regional rather than national level in settings where national malaria programmes interact, such as the Asia Pacific Malaria Elimination Network.

\section{Use case \#3: Assess transmission intensity}

This use case describes a method that allows decisionmakers to stratify regions according to transmission intensity in low and very low prevalence settings and monitor the effect of interventions. While current methods use surveys and weekly case counts to measure changes in prevalence and incidence, they are unable to measure genetic signatures indicative of changes in transmission intensity at low prevalence levels, such as changes in parasite population structure. For example, stagnation or increasing case counts might suggest an intervention is failing, even though the resurgence could be due to rising transmission rates from a source not exposed to the intervention. Additionally, for countries approaching elimination, this use case describes a method that can assess changes in transmission at low prevalence $(<2 \%)$, where prevalence estimates and case counts are imprecise due to geographical heterogeneity and are highly dependent on the population's access to health care. SNP barcodes have been used to define transmission patterns in populations in many countries, including Senegal [25], Malawi [26], Zambia [27], Cambodia [28], Panama [29], and through longitudinal tracking in Papua New Guinea [30]. Additional investigation is needed to advance and validate methods for the translation of parasite genetic diversity into a measurement of parasite transmission.

\section{Use case \#4: Identify foci}

This use case defines a method that enables the identification and stratification of foci according to receptivity and transmission intensity, as recommended by WHO 


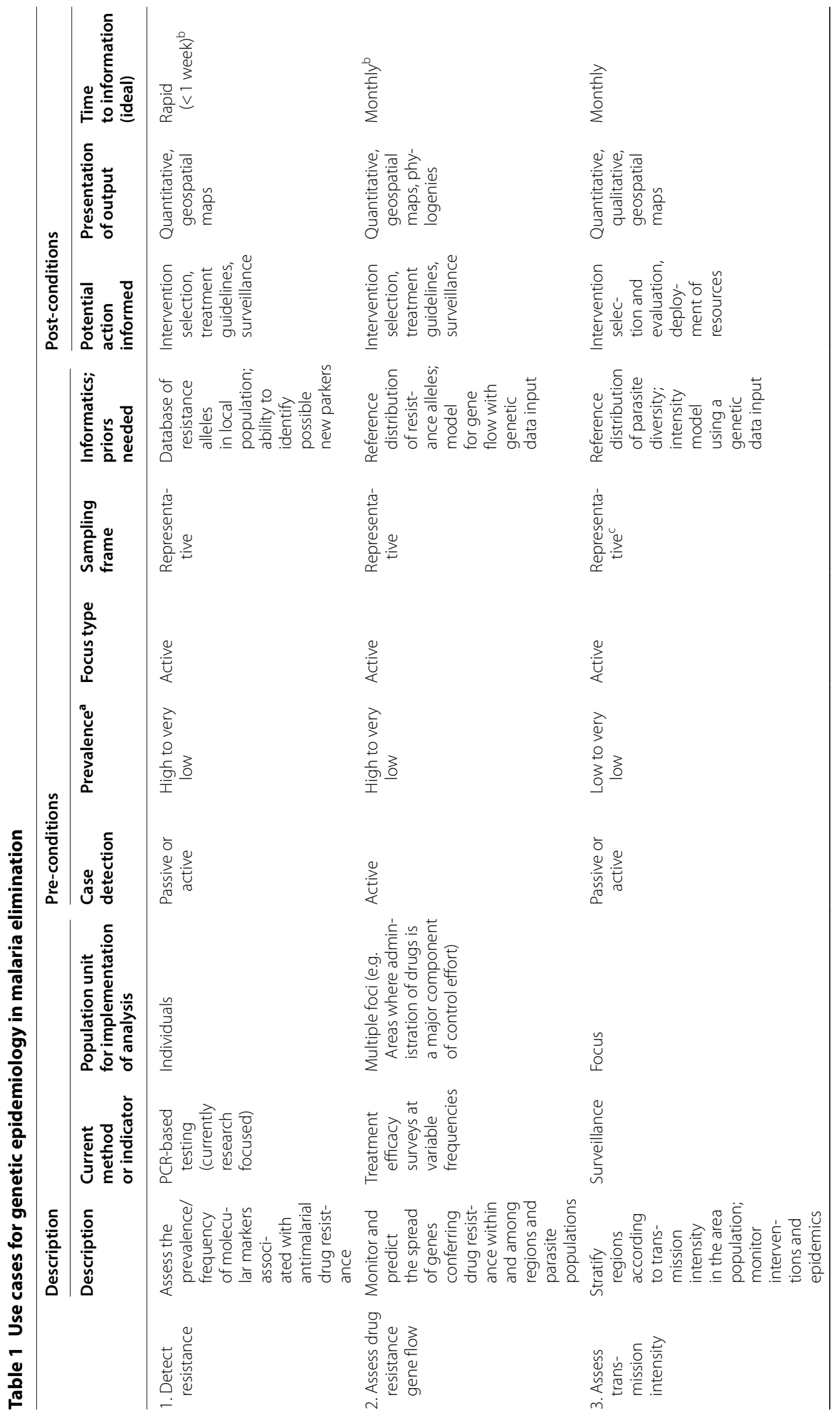




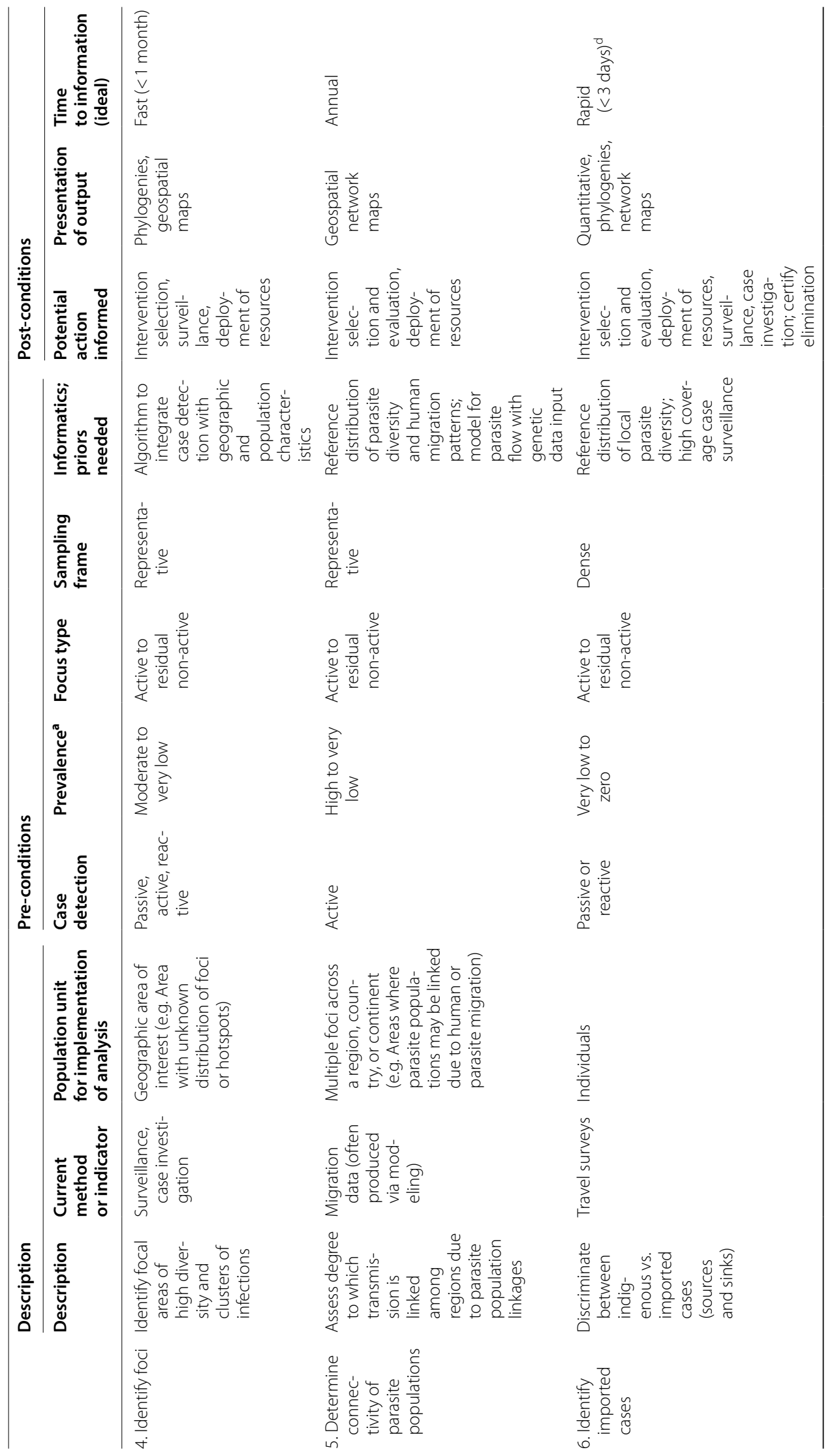




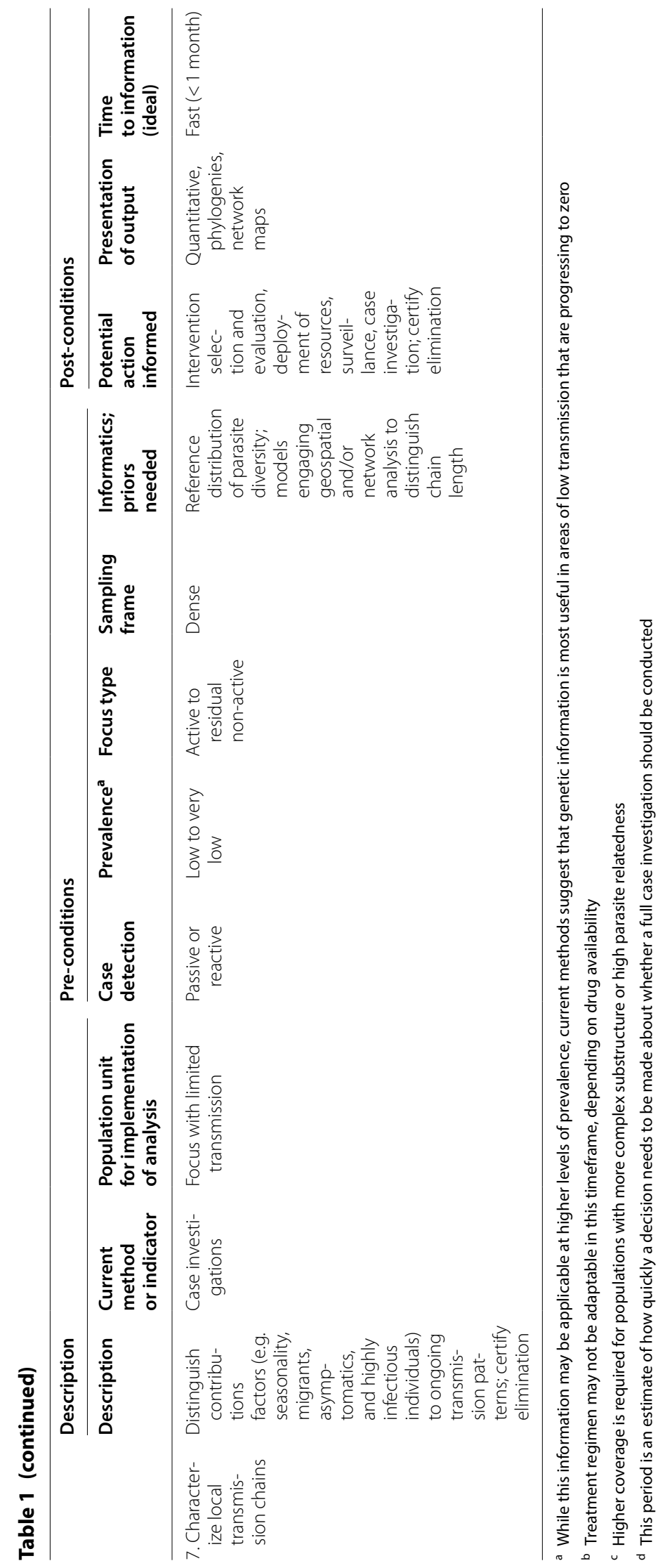


guidelines, in moderate to very low prevalence settings [12]. Specifically, the method measures how closely related parasites are to each other and estimates transmissibility of parasites within different groups. Currently, foci may be inferred using evidence from geospatial surveillance and case investigations, but only a genetic method can definitively link cases based on parasite genetic relatedness and therefore validate current methods for foci identification [31]. A method for identifying foci, especially in very low transmission settings, is likely to be linked to the method described by Use case 3 because transmission intensity is a characteristic used in foci stratification. In two examples, SNP-genotyping analysis generated by PCR-based assays was used to characterize the Makira region of Madagascar as a hotspot [32] and assays of microsatellites were used to attribute the resurgence of Plasmodium vivax malaria in Greece to specific villages with foci reactivated by imported malaria via migrant agricultural workers [33]. However, it is unclear what conditions and geographic scale are necessary to enable the identification of transmission foci that are stable over time using genetic surveillance.

\section{Use case \#5: Determine connectivity of parasite populations}

The method described in this use case determines how parasite populations are linked across various geographic regions with high to very low prevalence and enables a quantitative assessment of how much of transmission in one region is due to contributions from transmission in another. The method is related to the method presented in Use case 2, though without the specificity of anti-malarial resistance. Therefore, the two use cases may engage similar methods of measurement and analysis and are both relevant at a regional level. Estimates of how parasite populations are linked geospatially may be approximated from human migration data [34], but this use case proposes a method with higher spatiotemporal resolution. Gene flow has been characterized by a range of analytic techniques using SNP genotype data from nested PCR in Papua New Guinea [35] and at the Thai-Myanmar border [36], and genome-wide sequencing in Mauritania, among other countries [37]. However, these methods vary in their resolution to assert connectivity and additional evidence is needed to validate the associations between genetic patterns of population structure and human migration. An estimate of parasite population connectivity is an essential parameter for epidemiological models of malaria, which may be useful for optimizing elimination strategies [38].

\section{Use case \#6: Identify imported cases}

This use case describes a method for discriminating between indigenous and imported cases in areas with low or zero prevalence by measuring how similar a parasite is to a population of parasites currently or previously endemic to a geographically relevant area. In countries at or approaching zero cases, the method described in this use case provides a more definitive determination than can be offered through the use of travel surveys and case investigations. Several genetic methods have been demonstrated for this use cases in the literature, especially in countries at or approaching elimination that need to confirm whether a reported case or cluster of cases is due to local transmission or importation in order to maintain their elimination progress. Two high profile examples of such a method in use include: a cluster of cases in Puerto Rico confirmed to be imported from the Dominican Republic [39] and an outbreak of Plasmodium falciparum malaria among United Nations peacekeeping soldiers in Guatemala that was attributed to exposure when they were stationed in the Democratic Republic of Congo [40]. Optimization of these methods will be required to generate information useful in real-time scenarios.

\section{Use case \#7: Characterize local transmission chains}

The method described by this use case will be linked to the one proposed in Use case 6, but with higher resolution to distinguish the contribution of specific sources to ongoing or recurring transmission in an area with low to very low prevalence. The method will provide a high-resolution snapshot of how cases are related to each othera result definitively attainable only through a genetic method. Additionally, the method will need to incorporate both epidemiological and genetic information, as well as complex modeling methods to provide a snapshot of what is contributing to ongoing transmission. This use case is the most abstract in its conceptualization because methods for this purpose are mostly theoretical or have been modeled in specific research contexts [41]. Furthermore, a method for this use case will require a well characterized parasite population (historical and dense surveillance sampling) for the method to be applicable in that region. Few, if any, malaria endemic countries in the world have sufficiently historical and consistent genetic surveillance of their local parasite populations to contextualize a method for this use case.

\section{Conclusion}

The use cases presented in this article represent a novel application of a common business technique, applied elsewhere in fields of public health and software, and here to address the research-implementation gap in malaria. They have already been used to guide discussions at two international meetings focusing on the interactions between NMCP representatives and genome scientists, hosted by the Harvard T. Chan School of Public Health 
and the Massachusetts Institute of Technology Broad Institute (July 2017), and the Mahidol-Oxford Research Unit (January 2018).

Successful development and implementation of genetic epidemiological methods, through the steps depicted in Fig. 1, will require continued buy-in and information sharing between technical and programmatic experts, as well as resource investments that advance the capacity of NMCPs to involve these methods in their activities. This will be especially important in the translation of these use cases into the technical specifications needed for Target Product Profile (TPPs) that are directly responsive to specific NMCP information needs, including specific endemic settings and parasite-species specific requirements. NMCPs should drive the design of TPPs, and it is expected that different use cases will be prioritized by different countries and regions, depending on both epidemiological and operational contexts. While many of the use cases span a range of transmission intensities, there is a need to validate use cases, and the methods themselves, in higher burden settings. For example, routine monitoring for emergence of known antimalarial resistance markers in P. falciparum may be demanded by NMCPs in sub-Saharan Africa, while high resolution information about the spread of antimalarial resistance in $P$. vivax populations would be more useful in the Greater Mekong Subregion, Latin America, and other regions with endemic $P$. vivax. The development of TPPs may suggest opportunities for developing genetic epidemiology methods that address multiple use cases.

Additional implementation constraints and limitations of these new methods will also need to be explored in pragmatic test cases led by NMCPs to ensure these methods are cost effective in achieving elimination targets. Feasibility of each use case per country context will depend on factors including, but not limited to, the analytical capacity available, what sampling frame is logistically and financially feasible for ascertaining useful information at different levels of geographic granularity, the strength of the routine surveillance system, and availability and throughput capacity of laboratory technologies. There are pros and cons for NMCPs to use genetic approaches to answering epidemiological questions, and it is necessary to conduct a cost-benefit analysis for each use case. Significant involvement of programme experts and malaria-endemic country stakeholders will be required to help genetic technology developers understand where methods could be standardized for cost and supplement or perhaps even existing programme activities. This will help NMCPs understand whether existing epidemiological methods are sufficient in their country compared to opting for combining those methods with a genetic epidemiology approach, whether it is preferential to conduct this analysis in-country or in regional or global centers, and how best to integrate genetic epidemiology data into existing analysis for their decision-making [42].

It is worth noting that as this framework focuses on use cases applicable to questions NMCPs would ask, there are many more applications of genetic epidemiology for a research agenda than the use cases listed here. As one example, genotyping data can help us understand how long parasites persist, a critical element for assessing the infectious reservoir and from there for predicting the impact of interventions. In addition, having a representative sample of parasites globally would allow for prospective screening of drug resistance candidates to identify emerging resistance before countries start to observe treatment failure. A research agenda is beyond the scope of this article and would be a very welcome way of focusing efforts of the research community. Finally, these use cases can be extended to include vector genomics as the genomes of more vector species are sequenced and new methods emerge to detect and predict insecticide resistance emergence and spread.

By providing a framework for the application of genetic epidemiology approaches, these use cases can launch the process towards developing normative guidance for their appropriate use in global malaria eradication, a key element for any new tool or method to get to scale.

\section{Additional file}

Additional file 1. Technical and programmatic interview guides developed by the START center used in the semi-structured interviews with experts in the field.

\section{Abbreviations}

HRP2/HRP3: histidine-rich protein 2/3; NMCP: National Malaria Control Programme; NGS: next-generation sequencing; PCR: polymerase chain reaction; RDT: rapid diagnostic test; SDG: Sustainable Development Goals; SNP: single nucleotide polymorphism; TPP: Target Product Profile; WHO: World Health Organization.

\section{Authors' contributions}

Performed literature review, designed questionnaire and conducted interviews: RD, BN. Analysis and interpretation of data: RD, BN, JS. Drafting and revising manuscript: RD, BN, TSK, JS, EMS. Concept and design: EMS. All authors read and approved the final manuscript.

\footnotetext{
Author details

${ }^{1}$ Department of Epidemiology, University of Washington, Seattle, WA, USA ${ }^{2}$ Department of Global Health, University of Washington, Seattle, WA, USA. ${ }^{3}$ Department of Health Services, University of Washington, Seattle, WA, USA. ${ }^{4}$ Strategic Analysis Research and Training Center, University of Washington, Seattle, WA, USA. ${ }^{5}$ Bill and Melinda Gates Foundation, Seattle, WA, USA.
}

\section{Acknowledgements}

We thank Rachel Daniels, Bryan Greenhouse, Dan Hartl, Dominic Kwiatkowski, Bronwyn MacInnis, Olivo Miotto, Sean Murphy, Dan Neafsey, Rick Steketee, Wes VanVooris, Sarah Volkman, and Dyann Wirth for providing their expert 
input and feedback on the use cases at various stages of their development. We also thank Richard Maude, Francois Nosten, Carol Sibley, Venkatachalam Udhayakumar, and Edward Wenger for providing additional expertise in parasite biology, genetics, and diagnostics, as well as Mark Lim and Judd Walson for their input on the use case framework design. Our thanks also go to Maryska Valentine for her graphic design insights for Fig. 1.

\section{Competing interests}

Author Erin M. Stuckey is employed by the Bill \& Melinda Gates Foundation but had no role in the decision to fund the START Center.

\section{Availability of data and materials}

Not applicable.

\section{Consent for publication}

Not applicable.

\section{Ethics approval and consent to participate}

This research was reviewed by the University of Washington Human Subjects Division and determined to be exempt (IRB ID: STUDY00004708).

\section{Funding}

The Strategic Analysis, Research, and Training Center (START) at the University of Washington funded the original research (http://uwstartcenter.org/). START is a collaborative effort with, and is funded in part by, the Bill \& Melinda Gates Foundation (OPP1155935; https://www.gatesfoundation.org/).

\section{Publisher's Note}

Springer Nature remains neutral with regard to jurisdictional claims in published maps and institutional affiliations.

Received: 14 December 2018 Accepted: 22 April 2019

Published online: 07 May 2019

\section{References}

1. WHO. World malaria report 2017. Geneva: World Health Organization; 2017. http://www.who.int/malaria. Accessed 1 Feb 2018.

2. Khoury M, Beaty T, Cohen B. Fundamentals of genetic epidemiology. New York: Oxford University Press; 1993.

3. Auburn S, Barry AE. Dissecting malaria biology and epidemiology using population genetics and genomics. Int J Parasitol. 2017;47:77-85.

4. Greenhouse B, Smith DL. Malaria genotyping for epidemiologic surveillance. Proc Natl Acad Sci USA. 2015;112:6782-3.

5. WHO. False-negative RDT results and implications of new reports of P. falciparum histidine-rich protein 2/3 gene deletions. Geneva: World Health Organization; 2017. p. 1-11.

6. MalariaGEN. 2019. https://www.malariagen.net/. Accessed 2 Apr 2019.

7. Jacobson I. Use-Case 2.0 ebook. 2011. http://www.ivarjacobson.com/ Use_Case2.0_ebook/. Accessed 16 Mar 2017.

8. Lim MD, Brooker SJ, Belizario VY, Gay-Andrieu F, Gilleard J, Levecke B, et al. Diagnostic tools for soil-transmitted helminths control and elimination programs: a pathway for diagnostic product development. PLoS Negl Trop Dis. 2018;12:e0006213.

9. USAID. UAVs in Global Health defining a collective path forward. Washington: USAID; 2017.

10. MESA Alliance. MESA resource hub. 2019. https://mesamalaria.org/ resource-hub. Accessed 27 Jan 2017.

11. WHO. Global Malaria Programme. A framework for malaria elimination. Geneva: World Health Organization; 2017. p. 100. http://apps.who.int/ iris/bitstream/handle/10665/254761/9789241511988-eng.pdf?seque nce $=1$. Accessed 18 July 2017 .

12. WHO. Global technical strategy for malaria 2016-2030. Geneva: World Health Organization; 2015.

13. Mohon A, Menard D, Alam M, Perera K, Pillai D. A novel single-nucleotide polymorphism loop mediated isothermal amplification assay for detection of artemisinin-resistant Plasmodium falciparum malaria. Open Forum Infect Dis. 2018;5:ofy011.
14. Poschl B, Waneesorn J, Thekisoe O, Chutipongvivate S, Karanis P. Comparative diagnosis of malaria infections by microscopy, nested PCR, and LAMP in northern Thailand. Am J Trop Med Hyg. 2010;83:56-60.

15. Nag S, Dalgaard MD, Kofoed P-E, Ursing J, Crespo M, Andersen LO, et al. High throughput resistance profiling of Plasmodium falciparum infections based on custom dual indexing and illumina next generation sequencing technology. Sci Rep. 2017;7:2398.

16. Price RN, Auburn S, Marfurt J, Cheng Q. Phenotypic and genotypic characterisation of drug-resistant Plasmodium vivax. Trends Parasitol. 2012;28:522-9.

17. Rutledge G, Otto T. Last parasite standing. Nat Rev Microbiol. 2016;15:4.

18. Oriero E, Jacobs J, Van Geertruyden J-P, Nwakanma D, D'Alessandro $U$. Molecular-based isothermal tests for field diagnosis of malaria and their potential contribution to malaria elimination. J Antimicrob Chemother. 2015;70:2-13.

19. Roth J, Korevaar D, Leeflang M, Mens P. Molecular malaria diagnostics: a systematic review and meta-analysis. Crit Rev Clin Lab Sci. 2016;53:87-105.

20. Nsanzabana C, Djalle D, Guérin PJ, Ménard D, González IJ. Tools for surveillance of anti-malarial drug resistance: an assessment of the current landscape. Malar J. 2018;17:75.

21. Lo E, Hemming-Schroeder E, Yewhalaw D, Nguyen J, Kebede E, Zemene $\mathrm{E}$, et al. Transmission dynamics of co-endemic Plasmodium vivax and $P$. falciparum in Ethiopia and prevalence of antimalarial resistant genotypes. PLoS Negl Trop Dis. 2017;1 1:e0005806.

22. Dwivedi A, Khim N, Reynes C, Ravel P, Ma L, Tichit M, et al. Plasmodium falciparum parasite population structure and gene flow associated to anti-malarial drugs resistance in Cambodia. Malar J. 2016;15:319.

23. Baraka V, Delgado-Ratto C, Nag S, Ishengoma DS, Madebe RA, Mavoko HM, et al. Different origin and dispersal of sulfadoxine-resistant Plasmodium falciparum haplotypes between Eastern Africa and Democratic Republic of Congo. Int J Antimicrob Agents. 2017;49:456-64.

24. Talundzic E, Ravishankar S, Kelley J, Patel D, Plucinski M, Schmedes S, et al. Next-generation sequencing and bioinformatics protocol for malaria drug resistance marker surveillance. Antimicrob Agents Chemother. 2018;62:e02474-17.

25. Daniels RF, Schaffner SF, Wenger EA, Proctor JL, Chang H-H, Wong W, et al. Modeling malaria genomics reveals transmission decline and rebound in Senegal. Proc Natl Acad Sci USA. 2015;112:7067-72.

26. Sisya TJ, Kamn'gona RM, Vareta JA, Fulakeza JM, Mukaka MFJ, Seydel KB, et al. Subtle changes in Plasmodium falciparum infection complexity following enhanced intervention in Malawi. Acta Trop. 2015;142:108-14

27. Mharakurwa S, Daniels R, Scott A, Wirth DF, Thuma P, Volkman SK. Preamplification methods for tracking low-grade Plasmodium falciparum populations during scaled-up interventions in Southern Zambia. Malar J. 2014;13:89

28. Sluydts V, Heng S, Coosemans M, Van Roey K, Gryseels C, Canier L, et al. Spatial clustering and risk factors of malaria infections in Ratanakiri Province, Cambodia. Malar J. 2014;13:387.

29. Obaldia N, Baro NK, Calzada JE, Santamaria AM, Daniels R, Wong W, et al. Clonal outbreak of Plasmodium falciparum infection in Eastern Panama. J Infect Dis. 2015;211:1087-96.

30. Lerch A, Koepfli C, Hofmann NE, Kattenberg JH, Rosanas-Urgell A, Betuela I, et al. Longitudinal tracking and quantification of individual Plasmodium falciparum clones in complex infections. Sci Rep. 2019;9:3333.

31. Ruktanonchai NW, DeLeenheer P, Tatem AJ, Alegana VA, Caughlin TT, zu Erbach-Schoenberg E, et al. Identifying malaria transmission foci for elimination using human mobility data. PLoS Comput Biol. 2016;12:e1004846.

32. Rice BL, Golden CD, Anjaranirina EJG, Botelho CM, Volkman SK, Hartl DL. Genetic evidence that the Makira region in northeastern Madagascar is a hotspot of malaria transmission. Malar J. 2016;15:596.

33. Spanakos G, Snounou G, Pervanidou D, Alifrangis M, Rosanas-Urgell A, Baka A, et al. Genetic spatiotemporal anatomy of Plasmodium vivax malaria episodes in Greece, 2009-2013. Emerg Infect Dis. 2018;24:541-8.

34. Wesolowski A, Eagle N, Tatem AJ, Smith DL, Noor AM, Snow RW, et al. Quantifying the impact of human mobility on malaria. Science. 2012;338:267-70.

35. Fola AA, Nate E, Abby Harrison GL, Barnadas C, Hetzel MW, Iga J, et al. Nationwide genetic surveillance of Plasmodium vivax in Papua New Guinea reveals heterogeneous transmission dynamics and routes 
of migration amongst subdivided populations. Infect Genet Evol. 2018;58:83-95.

36. Taylor AR, Schaffner SF, Cerqueira GC, Nkhoma SC, Anderson TJC, Sriprawat K, et al. Quantifying connectivity between local Plasmodium falciparum malaria parasite populations using identity by descent. PLoS Genet. 2017;13:e1007065.

37. Duffy CW, Ba H, Assefa S, Ahouidi AD, Deh YB, Tandia A, et al. Population genetic structure and adaptation of malaria parasites on the edge of endemic distribution. Mol Ecol. 2017:26:2880-94.

38. Wesolowski A, Taylor AR, Chang H-H, Verity R, Tessema S, Bailey J, et al. Mapping malaria by combining parasite genomic and epidemiologic data. BMC Med. 2018;16:190.

39. Chenet SM, Silva-Flannery L, Lucchi NW, Dragan L, Dirlikov E, Mace K, et al. Molecular characterization of a cluster of imported malaria cases in Puerto Rico. Am J Trop Med Hyg. 2017;97:758-60.
40. Patel JC, Taylor SM, Juliao PC, Parobek CM, Janko M, Gonzalez LD, et al. Genetic evidence of importation of drug-resistant Plasmodium falciparum to Guatemala from the Democratic Republic of the Congo. Emerg Infect Dis. 2014;20:932-40.

41. Reiner RC, Le Menach A, Kunene S, Ntshalintshali N, Hsiang MS, Perkins TA, et al. Mapping residual transmission for malaria elimination. Elife. 2015;4:e09520.

42. Halsey ES, Venkatesan M, Plucinski MM, Talundzic E, Lucchi NW, Zhou Z, et al. Capacity development through the US President's malaria initiative-supported antimalarial resistance monitoring in Africa network. Emerg Infect Dis. 2017;23:13.
Ready to submit your research? Choose BMC and benefit from:

- fast, convenient online submission

- thorough peer review by experienced researchers in your field

- rapid publication on acceptance

- support for research data, including large and complex data types

- gold Open Access which fosters wider collaboration and increased citations

- maximum visibility for your research: over $100 \mathrm{M}$ website views per year

At BMC, research is always in progress.

Learn more biomedcentral.com/submissions 